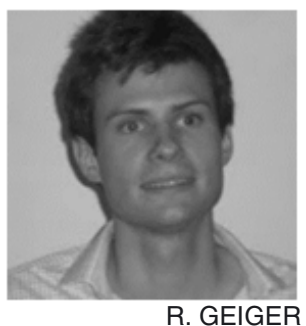

\title{
Gyromètre à atomes froids de grande sensibilité
}

\section{High sensitivity cold atom gyroscope}

\author{
Remi GEIGER, Indranil DUTTA, Denis SAVOIE, Bess FANG, Carlos L. GARRIDO ALZAR, \\ Christine GUERLIN, Matthieu MEUNIER, Thomas LEVEQUE, Bertrand VENON, Michel LOURS \\ et Arnaud LANDRAGIN
}

LNE-SYRTE, Observatoire de Paris, PSL Research University, CNRS, Sorbonne Universités, UPMC Univ. Paris 06, 61 avenue de l'Observatoire, 75014 Paris, France, remi.geiger@obspm.fr.

\begin{abstract}
Résumé
Nous présentons la nouvelle expérience de gyromètre à atomes froids du SYRTE mise en œuvre depuis 2009. Cette expérience utilise une configuration en fontaine atomique et un interféromètre atomique à quatre impulsions lumineuses pouvant permettre d'atteindre une aire Sagnac d'au moins $11 \mathrm{~cm}^{2}$. Nous avons démontré une stabilité de $3 \mathrm{nrad} \cdot \mathrm{s}^{-1}$ après $1000 \mathrm{~s}$ de temps d'intégration, ce qui représente l'état de l'art pour un gyromètre à atomes froids. Nous décrivons ici les éléments essentiels de l'expérience et les principales perspectives d'amélioration prévues à court et moyen terme.
\end{abstract}

$\underline{\text { MOTS CLÉS }}$ : ATOMES FROIDS, CAPTEUR INERTIEL, INTERFÉROMÉTRIE ATOMIQUE.

\begin{abstract}
We present the new cold atom gyroscope experiment developed at SYRTE since 2009. The experiment is based on a fountain configuration and a four light pulse atom interferometer which allows to reach a Sagnac area of at least $11 \mathrm{~cm}^{2}$. We demonstrated a gyroscope stability of $3 \mathrm{nrad} \cdot \mathrm{s}^{-1}$ after $1000 \mathrm{~s}$ of integration, which represents the state of the art for cold atom gyroscopes. Here we describe the main elements of the setup and the near future evolutions of the experiment to achieve better sensitivity levels.
\end{abstract}

KEYWORDS: COLD ATOMS, INERTIAL SENSOR, ATOM INTERFEROMETRY

\section{Introduction}

\subsection{Contexte}

Le développement de gyromètres de plus en plus performants représente un enjeu de recherche important tant les applications de ces capteurs sont importantes en navigation inertielle, en géophysique et en physique fondamentale. L'essor des gyromètres laser fondés sur l'effet Sagnac [1] à partir des années 1960 [2] a permis une révolution dans le domaine de la navigation inertielle. Cette technologie de capteurs s'est en effet imposée progressivement à partir des années 1980 dans les systèmes de navigation civils et militaires, en remplacement des centrales à plateformes stabilisées utilisant une plateforme à cardans ou des centrales à composants liés [3]. La stabilité à long terme des gyromètres laser modernes est comprise entre $10^{-2}{ }^{\circ} \cdot \mathrm{h}^{-1}$ et $10^{-3}{ }^{\circ} \cdot \mathrm{h}^{-1}$ [4] $\left(10^{-2}{ }^{\circ} \cdot \mathrm{h}^{-1} \approx 5 \times 10^{-8} \mathrm{rad} \cdot \mathrm{s}^{-1}\right)$. De meilleurs niveaux de stabilité dans la gamme des $10^{-4}{ }^{\circ} \cdot \mathrm{h}^{-1}$ ont par ailleurs été obtenus avec des gyromètres à fibre optiques, dont l'aire interférométrique est sous-tendue par une bobine de fibres optiques de longueur de l'ordre de $1 \mathrm{~km}$ [4]. Les gyromètres laser géants présentant une aire de plusieurs mètres carrés et des niveaux de sensibilités exceptionnels ont également permis des mesures directes des fluctuations de période de rotation de la Terre à des niveaux de l'ordre de $10^{-12} \mathrm{rad} \cdot \mathrm{s}^{-1}$ [5]. En comparaison, les gyroscopes supraconducteurs de la mission spatiale Gravity Probe B basés sur la détection du moment de London associé à la rotation d'un supraconducteur ont permis de mesurer avec une exactitude de 7 mas par an (correspondant à environ $1 \times 10^{-15} \mathrm{rad} \cdot \mathrm{s}^{-1}$ ) l'effet Lense-Thirring prédit par la Relativité Générale [6].

Le déphasage Sagnac dans un interféromètre présentant une aire physique orientée $\vec{A}$ est donné par

$$
\Delta \Phi=\frac{4 \pi E}{h c^{2}} \vec{\Omega} \cdot \vec{A}
$$




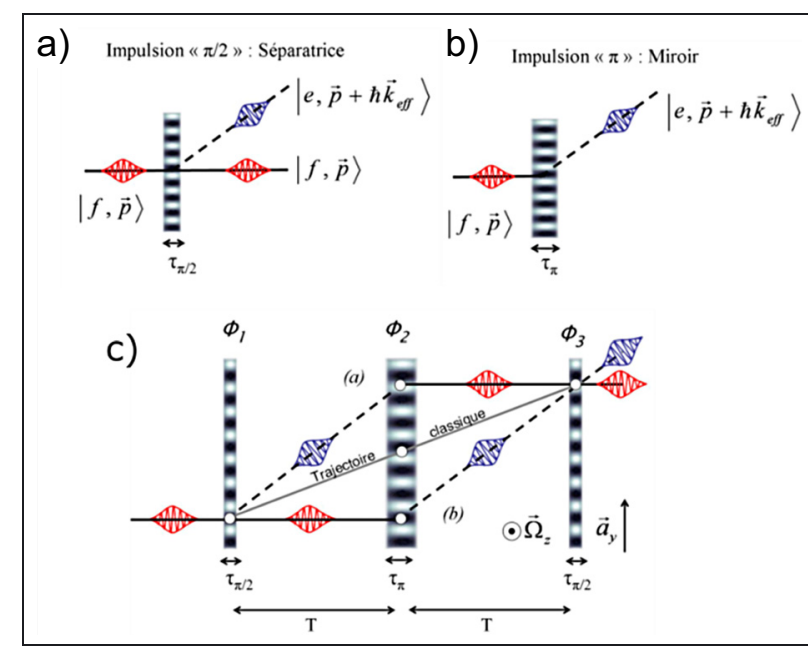

Fig. 1. - (a) Séparatrice et (b) miroir pour ondes de matière utilisant une interaction Raman à deux photons ; (c) interféromètre de type Mach-Zehnder à trois impulsions lumineuses; la durée entre deux impulsions Raman est notée $T$. Les figures sont extraites de la thèse de doctorat de Lévèque [7].

où $E$ représente l'énergie de la particule associée à l'onde se propageant dans l'interféromètre, $h$ la constante de Planck, $c$ la vitesse de la lumière et $\vec{\Omega}$ le vecteur de l'axe de rotation autour duquel l'interféromètre tourne par rapport à un référentiel d'inertie. L'utilisation d'ondes de matière pour lesquelles $E_{a t} \approx m c^{2}$ peut permettre d'obtenir des facteurs d'échelle du capteur beaucoup plus élevés que pour les gyromètres optiques pour lesquels l'énergie des photons $E_{p h}=h v$ est 11 ordres de grandeur inférieure à celle de particules massives, $E_{a t}$. Ainsi à aire interférométrique et sensibilité de phase équivalentes, un interféromètre à ondes de matière sera donc bien plus sensible aux rotations qu'un gyromètre optique. En pratique, la sensibilité de la mesure de phase dans un interféromètre optique est bien supérieure à celle d'un interféromètre atomique (due au flux de photons d'un laser environ 10 ordres de grandeur plus élevé que celui d'une source d'atomes froids). Les perspectives d'amélioration des capteurs atomiques sont cependant très intéressantes et nécessitent le développement de la technologie des atomes froids.

\subsection{Principe du gyromètre à atomes froids}

La réalisation d'interféromètres à ondes de matière nécessite l'équivalent des lames séparatrices et des miroirs utilisés en optique pour des ondes de matière. Plusieurs types de gyromètres atomiques ont été développés durant les vingt dernières années. Les meilleurs niveaux de sensibilité ont été obtenus dans des interféromètres utilisant des séparatrices pour ondes de matière fondées sur l'interaction d'un atome avec des lasers : l'interaction d'un atome à trois niveaux avec deux champs laser contre-propageant permet de créer une superposition cohérente de deux états d'impulsion différente de l'atome (fig. 1a et 1b). Ces deux états constituent les deux ondes se propageant suivant des chemins différents dans l'interféromètre (fig. 1c). L'interféromètre peut alors être réalisé dans le domaine spatial ou le domaine temporel. Dans le premier cas, un jet d'atomes est injecté dans l'interféromètre et la distance entre les séparatrices lasers détermine l'aire Sagnac. Dans le second cas, un nuage d'atomes froids est lancé dans une direction orthogonale à la direction de propagation des lasers et l'instant des impulsions séparatrices détermine l'aire Sagnac. Le gyromètre à atomes froids de grande sensibilité du SYRTE a été conçu suivant cette architecture, suite à l'ancienne version de l'expérience terminée en 2007 et présentée dans l'article de Landragin [8]. Plus de détails sur la comparaison des gyromètres à jet atomique et à atomes froids peuvent être trouvés dans l'article de Barrett [9].

Nous utilisons un schéma d'interaction de type transition Raman stimulées à deux photons pour des atomes de césium-133 : deux lasers dont la différence de fréquence correspond à l'écart en énergie entre les deux niveaux fondamentaux du césium permettent de créer des superpositions cohérentes de ces deux états avec des impulsions $\vec{p}$ et $\vec{p}+\hbar\left(\overrightarrow{k_{1}}-\overrightarrow{k_{2}}\right)$ où $\overrightarrow{k_{i}}$ désigne le vecteur d'onde du laser $i$. Dans le cas de deux lasers contre-propageant, de polarisations linéaires mutuellement orthogonales et dont un des faisceaux est obtenu par retro-réflexion sur un miroir, le transfert d'impulsion est égal en valeur absolue à $\hbar k_{\mathrm{eff}}=\hbar\left(k_{1}+k_{2}\right) \approx 2 \hbar k_{1}$, correspondant à une vitesse d'environ $7 \mathrm{~mm} \cdot \mathrm{s}^{-1}$ pour la transition optique à $852 \mathrm{~nm}$ du césium.

Le déphasage de l'interféromètre est donné par le déphasage Sagnac de l'équation (1). On peut montrer que ce déphasage atomique provient de la phase relative des deux lasers contre-propageant réalisant les séparatrices et miroirs, et celui-ci est imprimé sur la phase de la fonction d'onde atomique lors du processus d'interaction atome-champ laser [10]. Dans le cas d'un interféromètre à trois impulsions lumineuses (fig. 1c), la phase de sortie de l'interféromètre est alors donnée par $\Delta \Phi_{3 p}=\varphi_{1}-2 \varphi_{2}+\varphi_{3}$ où $\varphi_{i}$ est la différence de phase optique des deux lasers à l'instant de l'impulsion. La phase relative des deux lasers est essentiellement donnée par $\varphi(t)=\left(\overrightarrow{k_{1}}-\overrightarrow{k_{2}}\right) \cdot \vec{r}(t)$ où $\vec{r}(t)$ est la position de l'équiphase laser (déterminée par la position du miroir de rétroréflexion) par rapport au centre du nuage d'atomes en chute libre qui définit une référence d'inertie. Si le nuage d'atomes a une vitesse $\vec{v}$ dans l'interféromètre orientée perpendiculairement à la direction $\vec{k}_{\text {eff }}$ des lasers Raman, l'interféromètre possède alors une aire physique et est sensible à la rotation. Pour un interféromètre à trois impulsions Raman $\left(\frac{\pi}{2}-\pi-\frac{\pi}{2}\right)$, le déphasage est alors donné par $\Delta \Phi_{3 p}=\vec{k}_{\text {eff }} \cdot(2 \vec{\Omega} \times \vec{v}) T^{2}$ où $T$ est le temps entre les impulsions Raman. La sensibilité du capteur augmente donc avec le temps d'interrogation, qui est en pratique limité par la chute libre des atomes, i.e. la taille du dispositif.

Pour augmenter la sensibilité de l'interféromètre, un nouveau dispositif de gyromètre a été développé à partir de 2008 suivant une géométrie de fontaine atomique (fig. 2a). Les atomes sont lancés verticalement et une séquence de quatre impulsions Raman horizontales réalise l'interféromètre. La vitesse moyenne du nuage est alors 


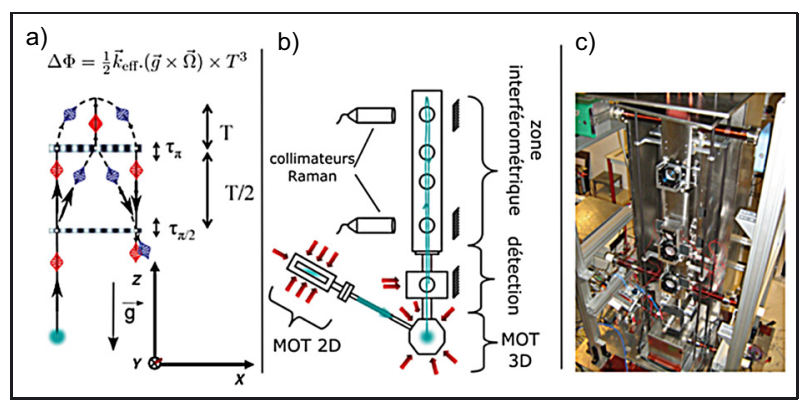

Fig. 2. - Schéma de l'expérience de gyromètre (a) trajectoire des atomes dans l'interféromètre à quatre impulsions Raman; (b) schéma de la chambre d'expérience montrant les principaux sous-systèmes; (c) photographie du système. La hauteur totale de la chambre à vide est 1,2 m. Le banc optique et l'électronique de contrôle ne sont pas sur la photographie.

donnée par le champ de pesanteur suivant $\vec{v}=\vec{g} t$, et le déphasage de l'interféromètre devient :

$$
\Delta \Phi_{4 p}=\frac{1}{2} \vec{k}_{\mathrm{eff}} \cdot(\vec{\Omega} \times \vec{g}) T^{3} .
$$

Pour un temps total d'interrogation $2 T=800 \mathrm{~ms}$ (le maximum accessible dans l'expérience), l'aire de l'interféromètre est égale à $11 \mathrm{~cm}^{2}$, donnant potentiellement accès à des sensibilités court terme de $10^{-9} \mathrm{rad} \cdot \mathrm{s}^{-1} / \sqrt{\mathrm{Hz}}$ pour un interféromètre limité par le bruit de projection quantique (bruit de grenaille atomique) pour $10^{6}$ atomes et un contraste de $20 \%$.

\section{Dispositif expérimental et performances du gyromètre}

\subsection{Dispositif expérimental}

Nous décrivons ici brièvement le dispositif expérimental, plus de détails pouvant être trouvés dans les thèses de doctorat de l'équipe $[7,11]$. Nous produisons dans un premier temps un nuage d'atomes froids préparé dans un piège magnéto-optique à trois dimensions (MOT 3D) en environ $200 \mathrm{~ms}$. Le nuage d'atomes est ensuite lancé verticalement à une vitesse ajustable entre $3,9 \mathrm{~m} \cdot \mathrm{s}^{-1}$ et $5 \mathrm{~m} \cdot \mathrm{s}^{-1}$ dans les expériences présentées dans cet article. Comme cette vitesse de lancement est très bien connue [7], nous savons exactement à quel moment le nuage arrive à l'apogée, et par conséquent, nous savons aussi exactement à quel instant le centre du nuage arrive à la zone de détection. En mesurant ce dernier paramètre nous déterminons la verticalité de la trajectoire des atomes. La température du nuage d'atomes après lancement est de $1,2 \mu \mathrm{K}$. Après une phase de préparation de l'état quantique initial des atomes à l'aide d'une transition micro-onde (paquet d'onde rouge dans la figure 2a se déplaçant vers le haut), ceux-ci sont soumis à une succession de quatre impulsions Raman $\left(\frac{\pi}{2}-\pi-\pi-\frac{\pi}{2}\right)$ réalisées en deux fenêtres différentes de la chambre de science (fig. 2b) (voir page 113 de la thèse de Lévèque [7]). Comme indiqué précédemment dans la figure 1 (partie 1.2), la première impulsion $\frac{\pi}{2}$ produit une superposition cohérente entre les deux états fondamentaux hyperfins du césium. Ainsi, le paquet d'onde

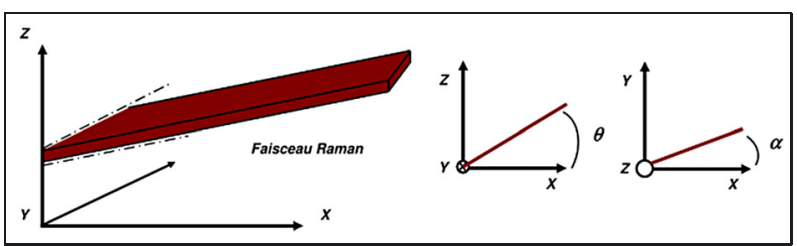

Fig. 3. - Définition des angles utilisés dans la procédure d'alignement des faisceaux Raman. Le plan $X Z$ correspond au plan de la figure 2 et l'alignement de chaque faisceau Raman dans ce plan sera décrit par un angle $\theta$, dans le plan perpendiculaire $X Y$, l'alignement sera décrit par l'angle $\alpha$.

bleu dans la figure 2a se déplaçant vers le haut, contient l'impulsion transmise par les faisceaux Raman. Après un temps de vol de durée $T / 2$, nous appliquons l'impulsion $\pi$ qui inverse les états interne et de propagation des atomes, et le paquet d'onde rouge devient bleu et vice versa. Ils font demi-tour à l'apogée et, après un temps de vol de durée $T$, ils sont soumis à une deuxième impulsion $\pi$. Les trajectoires s'inversent et la dernière impulsion $\frac{\pi}{2}$ est appliquée au moment de la rencontre des deux paquets d'onde.

La durée de l'interféromètre est de $2 T=480 \mathrm{~ms}$ et $2 T=800 \mathrm{~ms}$ dans les expériences que nous présentons ici. Par exemple, si $2 T=480 \mathrm{~ms}$, alors les impulsions $\frac{\pi}{2}$ et $\pi$ sont séparées de $120 \mathrm{~ms}$, et les deux impulsions $\pi$ sont séparées de $240 \mathrm{~ms}$. En fin de séquence expérimentale, l'état de sortie des atomes est détecté à l'aide d'un système de détection par fluorescence. Cette détection permet de déterminer la probabilité de transition $P$ d'un atome entre son état d'entrée dans l'interféromètre et son état de sortie, reliée au déphasage selon $P=P_{0}+B \cos \Delta \Phi$. Un ajustement de ce signal conduit à une détermination du déphasage, essentiellement dû à la rotation de la Terre.

La sensibilité de l'interféromètre aux rotations étant déterminée par le contraste des franges d'interférence, l'alignement relatif précis des lasers Raman issus des collimateurs dans la figure $2 \mathrm{~b}$ est un facteur clef pour atteindre les performances souhaitées de ce gyromètre atomique. En effet, s'il existe un écart angulaire $\delta \theta$ entre ces deux collimateurs dans le plan de la figure 2, alors l'interféromètre atomique ne se fermera pas car il existera un décalage vertical entre les deux nuages d'atomes au moment de la dernière impulsion qui est supposée recombiner ceux-ci. Si le décalage angulaire $\delta \alpha$ se situe dans le plan perpendiculaire à celui de la figure 2, alors il existera un décalage horizontal quand la dernière impulsion qui ferme l'interféromètre est appliquée. La conséquence étant, dans les deux cas, une perte de contraste de l'interféromètre liée à la longueur de cohérence finie de la source atomique et, par la suite, une perte de sensibilité de l'appareil. La définition des angles manipulés lors de l'alignement relatif des faisceaux Raman est présentée dans la figure 3 .

Chaque collimateur contient les raies optiques de pulsations $\omega_{1}$ et $\omega_{2}$ nécessaires à la réalisation des transitions Raman, de sorte qu'après rétro-réflexion on ait 
deux paires de faisceaux Raman par collimateur, un avec vecteur d'onde $+\vec{k}_{\text {eff }}$ et l'autre avec $-\vec{k}_{\text {eff }}$. Ainsi, la procédure que nous avons employée pour réaliser l'alignement de ces faisceaux comporte plusieurs étapes basées sur la réalisation d'interféromètres avec chaque collimateur. Dans un premier temps, le collimateur Raman inférieur dans la figure $2 \mathrm{~b}$ est positionné de sorte que le vecteur d'onde du faisceau sortant de celui-ci forme un angle de $94^{\circ}\left(=90^{\circ}+\theta\right)$ avec le vecteur vitesse $\vec{v}$ des atomes quand ceux-ci sont lancés vers la zone de l'interféromètre depuis le piège magnéto-optique MOT 3D. Cet angle permet, en utilisant l'effet Doppler, de lever la dégénérescence des transitions produites par $+\vec{k}_{\text {eff }}$ et $-\vec{k}_{\text {eff }}$. Ces deux transitions peuvent être observées en balayant le désaccord des lasers Raman autour de la fréquence d'horloge. Par ailleurs, la présence d'une transition à cette fréquence nous indique à quel point la polarisation des faisceaux Raman n'est pas linéaire car celle-ci correspond à une transition produite par des faisceaux co-propageant.

Pour que le collimateur supérieur fasse lui aussi un angle de $94^{\circ}$ avec le vecteur $\vec{v}$, de la même façon que le collimateur inférieur, nous avons utilisé le fait, qu'à cause de la gravité, la condition de résonance Raman évolue dans le temps comme $\vec{k}_{\text {eff }} \cdot \vec{g} t$. Nous avons alors appliqué une rampe de fréquence au laser Raman de sorte à compenser cette dépendance temporelle [11] qui produit un déphasage $\vec{k}_{\text {eff }} \cdot \vec{g} T^{2}$ à la sortie d'un interféromètre à trois impulsions. En utilisant exclusivement le collimateur inférieur, nous avons réalisé un interféromètre à trois impulsions $\left(\frac{\pi}{2}-\pi-\frac{\pi}{2}\right)$ afin de déterminer cette rampe dont sa valeur dépend de l'angle $\theta_{1}$ entre ce faisceau Raman et $\vec{v}$. Ensuite, nous avons utilisé la rampe ainsi déterminée pour annuler le déphasage lié à la gravité dans un interféromètre à trois impulsions réalisé avec le collimateur supérieur, en changeant l'angle $\theta_{2}$ que celui-ci forme avec $\vec{v}$. Avec cette procédure les deux collimateurs Raman ont été alignés avec un écart angulaire $\delta \theta=\theta_{1}-\theta_{2}$ de l'ordre de $2 \mu \mathrm{rad}$. Finalement, pour avoir un écart angulaire $\delta \alpha$ nul entre les deux collimateurs dans le plan $X Y$ dans la figure 3, nous avons optimisé le contraste d'un interféromètre Ramsey-Bordé [12] où les deux premières impulsions $\frac{\pi}{2}$ sont réalisées avec le collimateur inférieur et les deux dernières avec celui du haut. On peut montrer que l'écart angulaire maximum $\delta \alpha_{\max }$ entre les deux collimateurs est égal à :

$$
\delta \alpha_{\max }=\frac{L_{C}}{2 v_{r e c} d T},
$$

où $L_{C}=25 \mathrm{~nm}, v_{\text {rec }}$, et $d T$ sont respectivement la longueur de cohérence de la source atomique, la vitesse de recul et la séparation temporelle entre les deux impulsions réalisées dans chaque collimateur. La procédure d'alignement consiste alors à trouver le contraste maximum, pour une valeur $d T$ donnée, en changeant uniquement l'angle du collimateur supérieur dans la figure $2 b$. Ensuite, on augmente $d T$ et on cherche à nouveau à maximiser le contraste de l'interféromètre. En suivant cette procédure, nous avons obtenu un écart angulaire inférieur à $10 \mu \mathrm{rad}$ entre les deux collimateurs Raman dans le plan $X Y$ de la figure 3 .

\subsection{Réjection des vibrations et performances du gyromètre}

Outre sa sensibilité à la rotation de la Terre, l'interféromètre est sensible à tout effet inertiel impactant la position des deux miroirs de rétro-réflexion des lasers Raman. L'interféromètre est ainsi sensible aux accélérations des miroirs dans la direction $\vec{k}_{\text {eff }}$ des lasers, ainsi qu'aux rotations suivant la normale aux vecteurs $\vec{v}$ et $\vec{k}_{\text {eff }}$. Par conséquent, la phase $\Delta \Phi$ qui entre dans l'expression de la probabilité de transition $P$ (voir partie 2.1) contient, mise à part une contribution fixe de la vitesse de rotation de la Terre, une contribution liées aux variations rapides d'accélération. L'interféromètre n'opérant pas en continu et fournissant un signal inertiel moyenné sur une période correspondant aux temps d'interrogation des atomes, les variations rapides d'accélération ou de rotation se traduisent par du bruit sur le signal interférométrique. Typiquement, des variations d'accélération de $10^{-6} \mathrm{~m} \cdot \mathrm{s}^{-2}$ ou des variations de vitesse de rotation de $10^{-7} \mathrm{rad} \cdot \mathrm{s}^{-1}$ plus rapides que le temps d'interrogation $2 \mathrm{~T}$ se traduisent en un bruit de phase de l'ordre de $1 \mathrm{rad}$, nécessitant un contrôle du bruit de vibration. La fonction de sensibilité de l'interféromètre aux vibrations est maximale à la fréquence $\frac{2}{T} \approx 8 \mathrm{~Hz}$ (pour $2 T=480 \mathrm{~ms}$ ) et se comporte comme un filtre passe-bas aux fréquences supérieures [13]. En outre, l'interféromètre symétrique à quatre impulsions que nous utilisons n'est pas sensible aux accélérations constantes et se comporte comme un filtre passe-haut du premier ordre de fréquence de coupure $\frac{1}{T} \approx 4 \mathrm{~Hz}$. L'interféromètre est en revanche maximalement sensible au bruit de rotation de basse fréquence.

L'utilisation d'une plateforme anti-vibration passive nous permet de réduire de un à deux ordres de grandeur le bruit d'accélération dans la direction des faisceaux Raman pour des fréquences supérieurs à environ $0,5 \mathrm{~Hz}$, mais ne permet pas de réduire le bruit à plus basse fréquence. Pour réduire plus encore l'influence du bruit de vibrations, nous utilisons deux accéléromètres mécaniques (modèle Titan de la société Nanometrics) positionnés en haut et en bas de la chambre d'expérience, et combinons leurs signaux pour calculer l'influence du bruit d'accélération sur l'interféromètre, selon la méthode introduite dans [14]. Pour caractériser le gain apporté par cette méthode de rejection des vibrations, nous présentons dans la figure 4 le signal de sortie de l'interféromètre en fonction de la phase interférométrique calculée à partir des signaux accélérométriques. Le rapport signal à bruit, d'environ 12, est limité par le bruit résiduel de rotation qui n'est pas pris en compte dans ce traitement.

Pour déterminer la rotation de la Terre, nous ajustons les courbes de corrélation (interféromètre atomiqueaccéléromètres mécaniques) (comme présentée dans la fig. 4) par une sinusoïde, pour typiquement dix points de mesure. L'ajustement nous fournit la phase $\Phi_{\Omega}$ associée à la rotation de la Terre, et nous répétons cette procédure plusieurs fois (typiquement pour des centaines de paquets de dix points). Nous analysons la stabilité du gyromètre dans la figure 5 (courbe verte), qui présente l'écart type d'Allan des fluctuations de $\Phi_{\Omega}$. La courbe rouge présente 


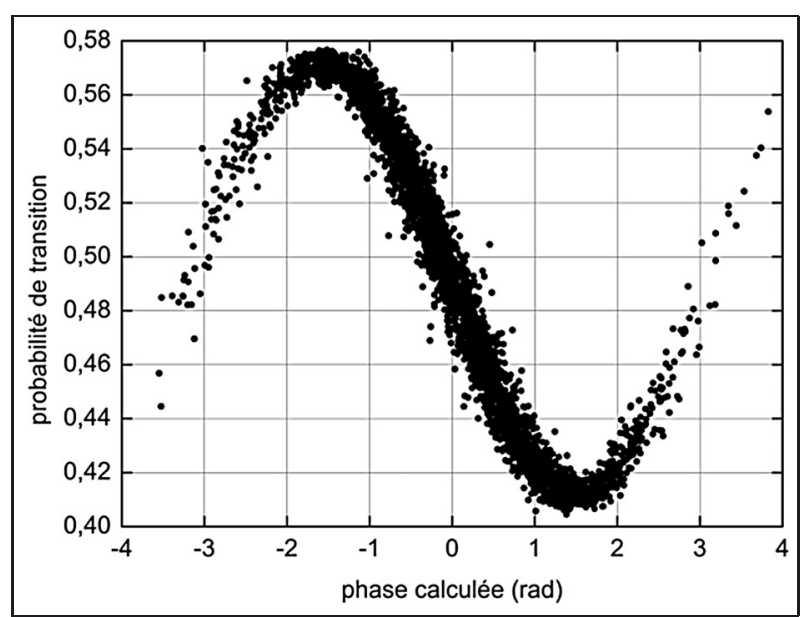

Fig. 4. - Signal de corrélation entre l'interféromètre atomique (probabilité de transition) et les accéléromètres mécaniques permettant d'estimer la phase interférométrique liée aux variations d'accélération (phase calculée). Ces données correspondent à un temps d'interrogation $2 T=480 \mathrm{~ms}$. Le rapport signal à bruit en environ égal à 12.

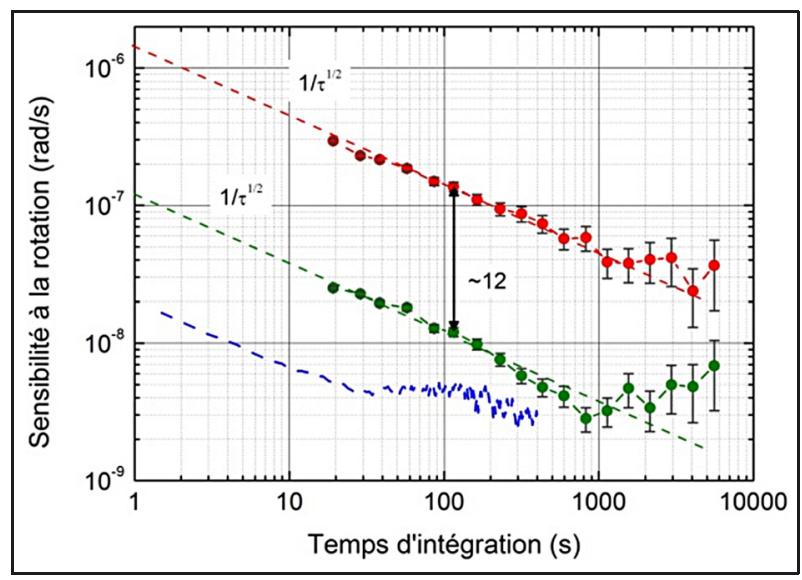

Fig. 5. - Stabilité du gyromètre pour $2 T=480 \mathrm{~ms}$; la courbe rouge (en haut) présente la stabilité sans réjection des vibrations avec les accéléromètres mécaniques ; la courbe verte (au milieu) donne la stabilité après réjection; la courbe bleue (en bas) correspond au bruit de détection.

la stabilité du gyromètre sans la réjection des vibrations avec les accéléromètres mécaniques. Nous retrouvons le facteur de réjection de 12 des vibrations, correspondant au rapport signal à bruit de la corrélation [interféromètreaccéléromètres]. La stabilité à court terme du gyromètre est de $120 \mathrm{nrad} \cdot \mathrm{s}^{-1}$, et est limitée par les bruits de vibration résiduels qui ne sont pas mesurés. La sensibilité s'intègre avec un comportement proche de $1 / \sqrt{\tau}$ jusqu'à environ $3 \mathrm{nrad} \cdot \mathrm{s}^{-1}$ après $1000 \mathrm{~s}$ de mesure, correspondant à une sensibilité de phase à long terme de l'interféromètre de $3 \mathrm{mrad}$. La dérive observée pour $\tau>1000 \mathrm{~s}$ est due aux fluctuations à long terme du déplacement lumineux qui n'est pas parfaitement rejeté dans la séquence expérimentale alternant les mesures suivant les deux vecteurs d'onde $\pm \vec{k}_{\text {eff }}$. La sensibilité à court terme que nous atteignons est vingt fois meilleure que celle obtenue par l'équipe de Stanford en 2011 [15] et est identique à celle

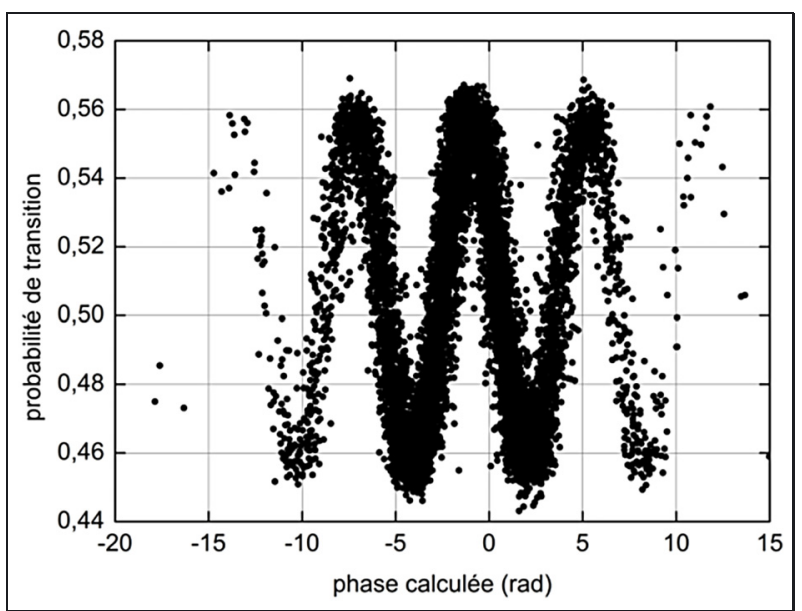

Fig. 6. - Signal de corrélation entre l'interféromètre atomique et les accéléromètres mécaniques pour un interféromètre d'aire égale à $11 \mathrm{~cm}^{2}$, c.-à-d. pour $2 T=800 \mathrm{~ms}$.

obtenue récemment par l'équipe de Hanovre [16]. La stabilité à long terme que nous obtenons est d'un ordre de grandeur meilleure que celle obtenue dans [16].

Les résultats précédents correspondent à un gyromètre d'aire égale à $2,4 \mathrm{~cm}^{2}(2 T=480 \mathrm{~ms})$. Pour augmenter encore la sensibilité du capteur, nous avons débuté des expériences avec le temps d'interrogation maximal $2 T=800 \mathrm{~ms}$, correspondant à une aire Sagnac de $11 \mathrm{~cm}^{2}$. Le facteur d'échelle du gyromètre (inverse du pré-facteur de $\vec{\Omega}$ de l'equation (2)) est alors de $4,5 \times 10^{6} \mathrm{rad} \cdot \mathrm{s}^{-1} / \mathrm{rad}$. Le signal de corrélation interféromètre-accéléromètres est présenté en figure 6. La plus grande sensibilité de l'interféromètre est claire, d'après l'excursion de phase calculée, comme étant environ quatre fois plus grande que dans le cas où $2 T=480 \mathrm{~ms}$ de la figure 4 . Dans ces résultats préliminaires, le rapport signal à bruit est ici limité par le bruit de détection associé au plus faible contraste de l'interféromètre à plus grand temps d'interrogation (10\% contre $17 \%)$, et par le bruit de rotation non pris en compte, de sorte que la sensibilité à court terme du gyromètre n'est pas encore améliorée par rapport à la configuration où $2 T=480 \mathrm{~ms}$.

L'amélioration de la sensibilité est en court. Au-delà du travail sur l'augmentation du contraste, nous mettons en œuvre actuellement un système de compensation en temps réel des vibrations, permettant de rester à flanc de frange de l'interféromètre en rétroagissant sur la phase des lasers Raman à partir du signal de phase calculée [17]. Un niveau de stabilité à $10000 \mathrm{~s}$ dans la gamme des $10^{-10} \mathrm{rad} \cdot \mathrm{s}^{-1}$ devrait être atteint d'ici la fin de l'année 2016.

\section{Mesures jointives}

\subsection{Motivation et principe}

Une limite actuelle importante des capteurs inertiels à atomes froids est leur fonctionnement séquentiel présentant un temps mort correspondant au temps de préparation et de détection des atomes. La perte d'information 
associée à ce temps mort limite les applications en navigation nécessitant une mesure continue. En outre, la présence de temps mort dégrade la sensibilité du capteur par un phénomène de repliement de spectre (analogue à l'effet Dick dans les horloges atomiques impactées par le bruit de l'oscillateur local).

Pour contourner ce problème, nous avons proposé une méthode d'interrogation sans temps mort dite jointive des capteurs inertiels à atomes froids [18]. Dans une architecture de fontaine atomique, il est possible de préparer un nuage d'atomes pendant que le nuage d'atomes précédent est interrogé dans l'interféromètre. En coordonnant le lancement d'un nouveau nuage avec l'arrivée du nuage précédent, il est possible d'annuler les temps morts et d'opérer le capteur avec une période de cycle égale au temps d'interrogation, $T_{c}=2 T$. Dans notre géométrie, cette configuration correspond au cas où la première séparatrice $\pi / 2$ du nuage entrant dans l'interféromètre à la montée a lieu au même instant que la séparatrice $\pi / 2 \mathrm{du}$ nuage sortant de l'interféromètre à la descente. En outre, en rendant simultanées les impulsions Raman d'interrogation, le bruit de vibration des lasers Raman est commun aux nuages d'atomes successifs. Les corrélations entre mesures successives peuvent donc permettre de moyenner le bruit de vibration plus efficacement qu'en $1 / \sqrt{\tau}$.

\subsection{Démonstration de principe de l'interrogation jointive en fonctionnement horloge}

Nous avons effectué une preuve de principe de l'interrogation jointive en utilisant notre expérience dans une configuration d'horloge, avec un interféromètre à deux impulsions Raman copropageantes $\pi / 2-\pi / 2$. Dans ce cas, l'interféromètre n'est pas sensible aux effets inertiels mais seulement au bruit de phase des lasers Raman qui jouent le rôle de l'oscillateur local d'une horloge. Le schéma de principe de l'interrogation jointive est donné en figure 7a. Nous montrons un moyennage efficace du bruit de l'oscillateur local en $1 / \tau$ associé aux corrélations entre mesures jointives de phase dans la figure $7 \mathrm{~b}$. Plus de détails sont donnés dans l'article de Meunier [18]. Dans un fonctionnement en mode capteur inertiel, bénéficier des corrélations entre mesures successives nécessite plus de deux nuages d'atomes dans l'interféromètre (trois nuages dans le cas d'un interféromètre à trois impulsions, quatre pour celui à quatre impulsions). En fonctionnement horloge, nous avons montré la possibilité d'un fonctionnement jointif allant jusqu'à cinq nuages d'atomes froids interrogés simultanément dans l'interféromètre, ainsi qu'une amélioration de la sensibilité court terme avec le nombre de nuages d'atomes [18]. Ces résultats laissent prévoir des perspectives d'amélioration significatives de la stabilité du gyromètre, pour atteindre des niveaux de $10^{-11} \mathrm{rad} / \mathrm{s}$ après $10000 \mathrm{~s}$ d'intégration.

\section{Conclusion}

Nous avons présenté le développement de la nouvelle expérience de gyromètre à atomes froids du SYRTE, mise

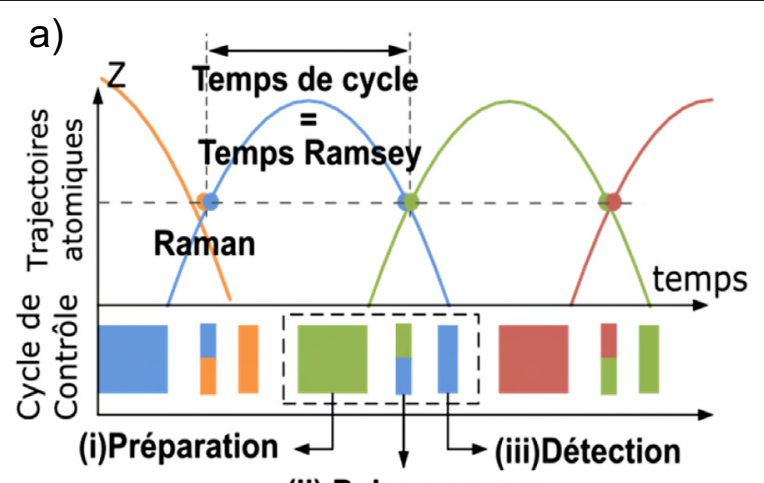

(ii) Pulse commun

b)

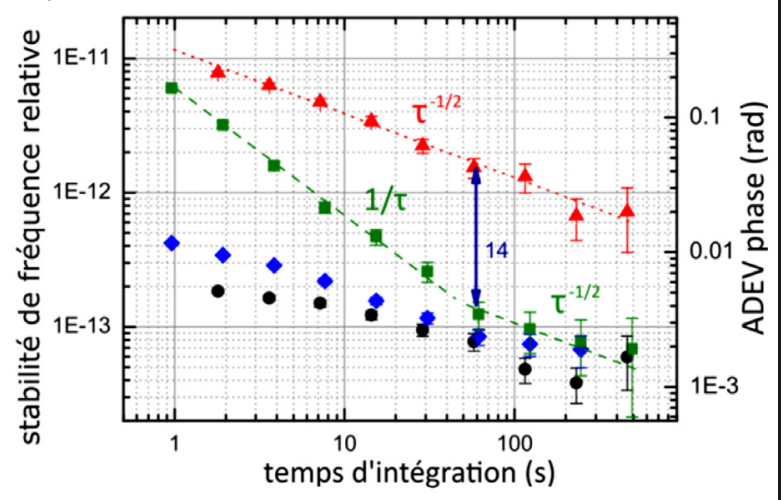

Fig. 7. - (a) Principe des mesures jointives en configuration horloge $\pi / 2-\pi / 2$. (b) Démonstration de la réjection du bruit de l'oscillateur local. La stabilité sans ajout de bruit est donnée par la courbe noire (cercles) en opération normale et par la courbe bleue (rhombes) en opération jointive. La stabilité avec ajout de bruit blanc sur une bande $400 \mathrm{~Hz}$ de largeur est donnée par la courbe rouge (triangles) en opération normale et par la courbe verte (carrés) en opération jointive (voir l'article de Meunier [18] pour plus de détails).

en œuvre depuis 2008. Cette expérience utilise une configuration en fontaine atomique et un interféromètre atomique à quatre impulsions lumineuses pouvant fonctionner avec un temps d'interrogation de $800 \mathrm{~ms}$, permettant d'atteindre une aire Sagnac d'au moins $11 \mathrm{~cm}^{2}$. Nous avons démontré une stabilité de $3 \mathrm{nrad} \cdot \mathrm{s}^{-1}$ après $1000 \mathrm{~s}$ de temps d'intégration, ce qui représente l'état de l'art pour un gyromètre à atomes froids. De nombreuses perspectives d'amélioration à court terme devraient permettre d'atteindre la gamme des $10^{-11} \mathrm{rad} \cdot \mathrm{s}^{-1}$ après $10000 \mathrm{~s}$ d'intégration. À moyen terme, des techniques de séparatrices multi-photoniques dans une cavité optique permettront d'augmenter le facteur d'échelle de l'interféromètre pour améliorer la sensibilité de l'instrument et atteindre la gamme des $10^{-12} \mathrm{rad} \cdot \mathrm{s}^{-1}$. Un fonctionnement doubleaxe du gyromètre est également à l'étude. De tels niveaux de sensibilité dans un instrument compact (par rapport au gyromètre laser géant) offriront des applications importantes en géophysique ou en physique fondamentale.

L'expérience de gyromètre du SYRTE permet le développement de nouvelles techniques d'interférométrie 
atomique sur un instrument représentant l'état de l'art en termes de sensibilité inertielle, et joue donc le rôle de démonstrateur pour d'autres expériences d'interférométrie atomique. En particulier, les recherches en amont menées sur l'expérience de gyromètre permettront de définir les évolutions de l'infrastructure MIGA (Matter-wave laser Interferometric Gravitation Antenna) [19], dont l'objectif est d'étudier à l'horizon 2018 le champ de gravité terrestre à l'aide d'un réseau d'interféromètres distribués sur un rayon de quelques centaines de mètres.

\section{Remerciements}

La réalisation de ces travaux a été possible grâce au soutien financier de la DGA, du CNES, de la Région Ile de France, du Labex FIRST-TF et de la Ville de Paris.

\section{Références}

[1] SAGNAC G., «L'éther lumineux démontré par l'effet du vent relatif d'éther dans un interféromètre en rotation uniforme », C. R. Acad. Sci. Paris, 95, 1913, 708-710.

[2] MaceK W.M. et DaVIS D.T.M., "Rotation Rate Sensing With Travelling-Wave Ring Lasers", Appl. Phys. Lett., 2 , 13, 1963, 67-68.

[3] Daussy Ch., «Le gyrolaser : Physique, technologies et production industrielle», chez SAGEM, présentation à l'institut d'Optique Graduate School, Palaiseau, 3 avril 2015.

[4] LEFÈVRE H.C., "The fiber-optic gyroscope, a century after Sagnac's experiment: The ultimate rotation-sensing technology ?", C. R. Phys., 15, 110, 2014, 851-858.

[5] Velikoseltsev A.A., LuK'yanov D.P., VinogRadov V.I. et SHREIBER K.U., "Super-large optical gyroscopes for applications in geodesy and seismology: state-of-theart and development prospects", Quantum Electron., 44, $112,2014,1151-1156$.

[6] EveritT C.W.F. et al., "Gravity Probe B: Final Results of a Space Experiment to Test General Relativity", Phys. Rev. Lett., 106, 122, 2011, 221101.

[7] LÉVÈQUE T., « Développement d'un gyromètre à atomes froids de haute sensibilité fondé sur une géométrie repliée », http://www.theses.fr/2010PA066298, 2010.

Article reçu le 6 août 2015, version révisée reçue le 18 février 2016.
[8] Landragin A., Canuel B., Gauguet A. et Tuckey $\mathrm{Ph}$., «Capteur inertiel six axes fondé sur l'interférométrie atomique », Revue française de métrologie, 10, 2007, 11-16.

[9] Barrett B., Geiger R., Dutta I., Meunier M., Canuel B., Gauguet A., Bouyer P. et Landragin A., "The Sagnac effect: 20 years of development in matterwave interferometry", C. R. Phys., 15, 110, 2014, 875883.

[10] BORDÉ Ch., "Quantum Theory of Atom-Wave Beam Splitters and Application to Multidimensional Atomic Gravito-Inertial Sensors", Gen. Relativity Gravitation, 36, 13, 2004, 475-502.

[11] Meunier M., "Étude d'un gyromètre à ondes de matière de très grande aire », http://www.theses.fr/ 2013PA066660, 2013.

[12] BORDÉ Ch., "Atomic interferometry with internal state labelling", Phys. Lett. A, 140, 11, 1989, 10-12.

[13] Cheinet P., Canuel B., Pereira Dos Santos F., GAUGUET A., LEDUC F. et LANDRAGIN A., "Measurement of the sensitivity function in time-domain atomic interferometer", IEEE Trans. Instrum. Meas., 57, 2008, 1141-1148.

[14] Merlet S., Le Gouët J., Bodart Q., Clairon A., LANDragin A., Pereira Dos SANTos F. et Rouchon, P., "Operating an atom interferometer beyond its linear range", Metrologia, 46, 11, 2009, 87-94.

[15] Stockton J.K., Takase K. et Kasevich M.A., "Absolute Geodetic Rotation Measurement Using Atom Interferometry", Phys. Rev. Lett., 107, 113, 2011, 133001.

[16] Berg P., Abend S., Tackmann G., Schubert C., GIESE E., SCHLEICH W.P.,. NARDUCCI F.A., ERTMER W. et RASEL E.M., "Composite-Light-Pulse Technique for High-Precision Atom Interferometry", Phys. Rev. Lett., 114, 2015, 063002.

[17] Lautier J., Volodimer L., Hardin T., Merlet S., Lours M., Pereira Dos SANTOS F. et LANDRAGin A., "Hybridizing matter-wave and classical accelerometers", Appl. Phys. Lett., 105, 114, 2014, 144102.

[18] Meunier M., Dutta I., Geiger R., Guerlin C., GARRIDO AlZAR C.L. et LANDRAGIN A., "Stability enhancement by joint phase measurements in a single cold atomic fountain", Phys. Rev. A, 90, 2014, 063633.

[19] Geiger R., Amand L., Bertoldi A., Canuel B., Chaibi W., Danquigny C., Dutta I., Fang B., GafFet S., Gillot J., Holleville D., LANDRagin A., Merzougui M., Riou I., Savoie D., Bouyer P., "Matter-wave laser Interferometric Gravitation Antenna (MIGA): New perspectives for fundamental physics and geosciences", Proceedings of the 50th Rencontres de Moriond "100 years after GR", La Thuile, Italie, 21-28 mars 2015, arXiv: 1505.07137, 2015. 